\title{
Response to: an easy insertion procedure of Bakri balloon during cesarean section for placenta previa: use of Nelaton rubber catheter
}

\author{
Baris Kaya
}

Published online: 29 July 2014

(c) Springer-Verlag Berlin Heidelberg 2014

Bakri balloon is a very useful device in the management of postpartum hemorrhage which was originally produced for placenta previa cases. I agree with Matsubara [1] that removing stopcock part of the dual end vaginal portion of the Bakri balloon is useful while passing through the narrow cervix during cesarean section. Matsubara's new method can be used in the placement of the Bakri balloon in the placenta previa with/without accreta cases and also in uterine atony cases, especially which have a narrow or nearly closed cervix ones.

Conflict of interest None.

\section{Reference}

1. Matsubara S (2014) An easy insertion procedure of Bakri balloon during cesarean section for placenta previa: use of Nelaton rubber catheter. Arch Gynecol Obstet. doi:10.1007/s00404-014-3380-8 